\title{
An Algorithm for Estimating the Signal Frequency at the Output of a Channel with a Controlled Information Flow under Phase Noise Conditions
}

\author{
L. N. Kazakov ${ }^{1}$, E. P. Kubyshkin ${ }^{1}$, I. V. Lukyanov ${ }^{2}$ \\ ${ }^{1}$ P. G. Demidov Yaroslavl State University, 14 Sovetskaya str., Yaroslavl 150003, Russia. \\ ${ }^{2}$ OOO “NPP “LAMA” ”, 89 Serova str, Rybynsk 152907, Russia.
}

DOI: $10.18255 / 1818-1015-2021-4-452-461$

MSC2020: 68W25, 68W40

Research article

Full text in Russian
Received November 15, 2021

After revision December 1, 2021

Accepted December 8, 2021

Research in the field of efficient frequency estimation algorithms is of great interest. The reason for this is the redistribution of the role of additive and phase noise in many modern radio-engineering applications. An example is the area of measuring radio devices, which usually operate at high signal-to-noise ratios (SNR). The estimation error is largely determined not by the broadband noise, but by the frequency and phase noise of the local oscillators of the receiving and transmitting devices. In particular, earlier works [1] proposed an efficient computational algorithm for estimating the frequency of a quasi-harmonic signal based on the iterative calculation of the autocorrelation sequence (ACS). In [2], this algorithm was improved and its proximity to the Rao-Cramer boundary was shown (the sources of this noise are master oscillators and frequency synthesizers). Possibilities of frequency estimation in radio channels make it possible to significantly expand the functionality of the entire radio network. This can include, for example, the problem of adaptive distribution of information flows of a radio network. This also includes the tasks of synchronization and coherent signal processing. For these reasons, more research is needed on this algorithm, the calculation of theoretical boundaries and their comparison with the simulation results.

Keywords: Rao-Cramer boundary; autocorrelation function; frequency instability; phase noise; Fisher information

INFORMATION ABOUT THE AUTHORS

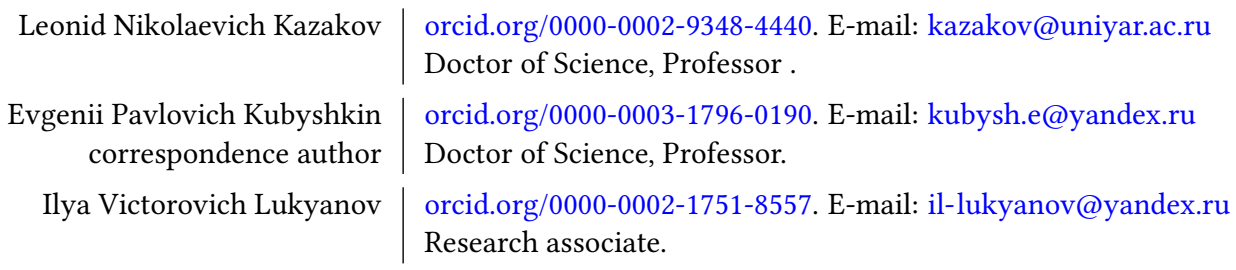

Funding: The reported study was funded by YSU Programme according to the research project № P2-K-1-G-4/2021.

For citation: L. N. Kazakov, E. P. Kubyshkin, and I. V. Lukyanov, "An Algorithm for Estimating the Signal Frequency at the Output of a Channel with a Controlled Information Flow under Phase Noise Conditions", Modeling and analysis of information systems, vol. 28, no. 4, pp. 452-461, 2021. 


\title{
Алгоритм оценивания частоты сигнала на выходе канала с управляемым информационным потоком в условиях фазового шума
}

\author{
Л. Н. Казаков ${ }^{1}$, Е. П. Кубышкин ${ }^{1}$, И. В. Лукьянов ${ }^{2}$
}

DOI: $10.18255 / 1818-1015-2021-4-452-461$

${ }_{1}^{1}$ Ярославский государственный университет им. П. Г. Демидова, ул. Советская, д. 14, г. Ярославль, 150003 Россия. ${ }^{2} \mathrm{OOО}$ «НПП «ЛАМА», пр-т Серова, д. 89, г. Рыбинск, 152907 Россия.

УДК 519.7, 621.396 .96

Научная статья

Полный текст на русском языке
Получена 15 ноября 2021 г.

После доработки 1 декабря 2021 г. Принята к публикации 8 декабря 2021 г.

Большой интерес вызывают исследования в области эффективных алгоритмов оценивания частоты. Причиной этому является перераспределение во многих современных радиотехнических приложениях роли аддитивного и фазового шумов. Примером может служить область измерительных радиоприборов, работающих, как правило, при высоких отношениях сигнал/шум (ОСШ). Ошибка оценки в большей степени определяется не широкополосным шумом, а частотными и фазовыми шумами гетеродинов приемных и передающих устройств. В частности, в более ранних работах [1] предложен эффективный вычислительный алгоритм оценивания частоты квазигармонического сигнала, основанный на итерационном вычислении АКП. В [2] этот алгоритм доработан и показана его близость к границе Рао-Крамера (источниками этих шумов являются задающие генераторы и синтезаторы частоты). Возможности оценивания частоты в радиоканалах позволяют существенно расширить функционал всей радиосети. Сюда можно отнести, например, задачу адаптивного распределения информационных потоков радиосети. Сюда же можно отнести задачи синхронизации и когерентной обработки сигналов. По этим причинам необходимы дополнительные исследования этого алгоритма, расчет теоретических границ и их сравнение с результатами моделирования.

Ключевые слова: граница Рао-Крамера; автокорреляционная функция; частотная нестабильность; фазовый шум; информация Фишера

\section{ИНФОРМАЦИЯ ОБ АВТОРАХ}

Леонид Николаевич Казаков

Евгений Павлович Кубышкин автор для корреспонденции Илья Викторович Лукьянов orcid.org/0000-0002-9348-4440. E-mail: kazakov@uniyar.ac.ru доктор технических наук, профессор.

orcid.org/0000-0003-1796-0190. E-mail: kubysh.e@yandex.ru доктор физико-математических наук, профессор.

orcid.org/0000-0002-1751-8557. E-mail: il-lukyanov@yandex.ru научный сотрудник .

Финансирование: Исследование выполнено в рамках Программы развития ЯрГУ, проект № П2-К-1-Г-4/2021.

Для цитирования: L. N. Kazakov, E.P. Kubyshkin, and I. V. Lukyanov, "An Algorithm for Estimating the Signal Frequency at the Output of a Channel with a Controlled Information Flow under Phase Noise Conditions", Modeling and analysis of information systems, vol. 28, no. 4, pp. 452-461, 2021.

(с Казаков Л.Н., Кубышкин Е. П., Лукьянов И. В., 2021

Эта статья открытого доступа под лицензией СС BY license (https://creativecommons.org/licenses/by/4.0/). 


\section{Введение}

В [1] предложен эффективный вычислительный алгоритм оценивания частоты квазигармонического сигнала, основанный на итерационном вычислении АКП. В [2] этот алгоритм доработан и показана его близость к границе Рао-Крамера (ГРК). В этих публикациях, как и в большинстве других известных работ, посвященных алгоритмам оценивания частоты, представлены результаты моделирования в условиях аддитивного белого гауссовского шума (АБГШ). Однако в измерительных радиосистемах, работающих, как правило, при высоких отношениях сигнал/шум (ОСШ), ошибка оценки в большей степени определяется не широкополосным шумом, а частотными и фазовыми шумами гетеродинов приемных и передающих устройств. Источниками этих шумов являются задающие генераторы и синтезаторы частоты [3]. По этим причинам необходимы дополнительные исследования этого алгоритма, расчет теоретических границ и их сравнение с результатами моделирования.

\section{1. МОДЕЛИ ФАЗОВОГО ШУМА}

Модель комплексного сигнала на выходе канала с АБГШ и фазовым шумом можно записать в виде

$$
s(t)=A \exp \left(j\left[2 \pi f_{0} t+\varphi(t)\right]\right)+\dot{w}(t),
$$

где $t$ - время; $A$ - амплитуда; $f_{0}$ - номинальная частота; $\varphi(t)$ - фазовая функция сигнала; $\dot{w}(t)-$ АБГШ.

Под частотным и фазовым шумами понимаются функции:

$$
\Delta f(t)=f_{0}-\hat{f}(t), \Delta \phi(t)=\varphi(t)-\hat{\varphi}(t),
$$

где $\hat{f}(t), \hat{\varphi}(t)$ - фактические мгновенные значения частоты и фазы сигнала.

Частотные и фазовые шумы генераторов характеризуются следующими функциями $[3,4]$ :

- спектральная плотность фазовых флуктуаций

$$
S_{\varphi}(f)=\lim _{T \rightarrow+\infty} \frac{2}{T}\left|\int_{T / 2}^{T / 2} \Delta \varphi(t) \exp (-j 2 \pi f t) d t\right|^{2} ;
$$

- спектральная плотность частотных флуктуаций

$$
S_{F}(f)=\lim _{T \rightarrow+\infty} \frac{2}{T}\left|\int_{T / 2}^{T / 2} \Delta f(t) \exp (-j 2 \pi f t) d t\right|^{2}=f^{2} S_{\varphi}(f) ;
$$

- спектральная плотность относительного отклонения частоты

$$
S_{Y}(f)=\left(\frac{f}{f_{0}}\right)^{2}, S_{\varphi}(f)=\left(\frac{1}{f_{0}}\right)^{2} S_{F}(f),
$$

где $T$ - интервал наблюдения.

К интегральным характеристикам частотной нестабильности относятся паразитные отклонения фазы (ПОФ) и паразитные отклонения частоты (ПОЧ), определяемые выражениями:

$$
\sigma_{\varphi}\left(f_{H}, f_{B}\right)=\sqrt{\int_{f_{H}}^{f_{B}} S_{\varphi}(f) d f}, \sigma_{f}\left(f_{H}, f_{B}\right)=\sqrt{\int_{f_{H}}^{f_{B}} S_{f}(f) d f} .
$$


Общепризнанной моделью представления спектральной плотности фазовых и частотных флуктуаций является степенная модель [3]:

$$
S_{M}(f)=\sum_{a} h_{a} f^{a}
$$

где $a$ характеризует вид шума; $h_{a}$ характеризует мощность шума.

В таблице 1 представлена классификация основных частотных и фазовых шумов генераторов $[3,4]$.

Table 1. Classification of frequency and phase

Таблица 1. Классификация частотного

\begin{tabular}{|l|c|c|}
\hline \multirow{2}{*}{\multicolumn{2}{|c|}{ Тип шуме }} & \multicolumn{2}{c|}{ и фазового шума } \\
\cline { 2 - 3 } & $S_{Y}(f)=h_{a} f^{a}$ & $S_{\varphi}(f)=f_{0}^{2} h_{\beta} f^{\beta}$ \\
\cline { 2 - 3 } & $\mathrm{a}$ & $\beta=\mathrm{a}-2$ \\
\hline Частотный шум случайных блужданий & -2 & -4 \\
\hline Частотный фликкер-шум & -1 & -3 \\
\hline Белый частотный шум & 0 & -2 \\
\hline Фазовый фликкер-шум & 1 & -1 \\
\hline Белый фазовый шум & 2 & 0 \\
\hline
\end{tabular}

\section{2. АНАЛИЗ ТЕОРЕТИЧЕСКИХ ГРАНИЦ АЛГОРИТМА}

Представим дискретную модель сигнала в условиях фазового шума и АБГШ в виде

$$
x[n]=A \exp (j \varphi[n]) \exp \left(j\left[2 \pi f_{0} n+\varphi_{0}\right]\right)+\dot{w}[n],
$$

где $\mathrm{n}=0,1, \ldots, N \lambda 1$ - номер отсчета; $N$ - размер выборки; $\varphi[n]$ - отсчеты фазового шума; $f_{0}$ нормированная номинальная частота; $\varphi_{0}$ - начальная фаза; $\dot{w}[n]-$ комплексные отсчеты АБГШ с дисперсией $\sigma_{w}^{2}$.

При исследовании алгоритмов оценивания интерес представляет сравнение среднеквадратической ошибки (СКО) оценки с некоторой теоретической границей. При этом СКО оценки определяется в соответствии с выражением

$$
\varepsilon=\sqrt{\frac{1}{K} \sum_{i=0}^{K-1}\left(\hat{f}_{i}-f_{0}\right)^{2}},
$$

где $K$ - число экспериментов; $\hat{f}_{i}$ - оценка нормированной частоты.

Общепризнанным количественным критерием алгоритмов оценивания является сравнение их СКО с границей Рао-Краммера (ГРК). Анализ публикаций показал, что на сегодняшний день для модели (1) в аналитическом виде (с рядом допущений) ГРК получена только для случая, когда $\varphi[n]$ является нормальным процессом [5]. Математические сложности при выводе ГРК обусловлены нестационарностью процессов, классифицированных в таблице 1, и невозможностью аналитического представления их автокорреляционных функций.

Рассмотрим эту проблему подробнее. Будем считать, что $\varphi[n]$ в общем случае является нестационарным процессом с ковариационной матрицей $\mathbf{Q}_{\varphi}[n]$ и математическим ожиданием $\mu_{\varphi}[n]$, где $\mathrm{n}$ означает зависимость статистических параметров от времени наблюдения.

ГРК в общем случае определяется элементами главной диагонали обратной матрицы Фишера [6] с элементами 


$$
[I(\Theta)]_{i j}=\left[\frac{\partial \mu(\Theta)}{\partial \Theta_{i}}\right]^{T} Q^{-1}(\Theta)\left[\frac{\partial \mu(\Theta)}{\partial \Theta_{j}}\right]+\frac{1}{2} \operatorname{tr}\left[Q^{-1}(\Theta) \frac{\partial Q(\Theta)}{\partial \Theta_{i}} Q^{-1}(\Theta) \frac{\partial Q(\Theta)}{\partial \Theta_{j}}\right],
$$

где $\Theta$ - вектор оцениваемых параметров; $\mu$ и $\Theta$ - математическое ожидание и ковариационная матрица процесса (1).

Для нестационарных процессов $\varphi[n]$ (когда показатель $\beta<0$ ) аналитический расчет по (3) невозможен. Для этого требуется представление автокорреляционной функции процесса $\varphi[n]$ в стационарном приближении, что ставит под сомнение корректность полученного критерия.

\section{А. Приближенный расчет СКО оценки по спектральной плотности фазового шума}

Другим путем получения теоретического критерия, является использование выражений, представленных в работе [7]. Считая аддитивный шум и фазовые флуктуации независимыми процессами, а измерительную систему линейной, такой показатель можно записать в виде

$$
\sigma_{\hat{f}}^{2}=\frac{N_{I}}{N_{I}-1} \int_{f_{H}}^{f_{B}} S_{\phi}(f) \frac{\sin ^{2}(\pi \tau f)}{(\pi \tau)^{2}}\left[1-\frac{\sin ^{2}\left(T_{\Pi} \pi f N_{I}\right)}{N_{I}^{2} \sin ^{2}\left(T_{\Pi} \pi f\right)}\right] d f+\frac{6 f_{\partial}}{(w \pi)^{2} R_{l} \tau\left(\left(\tau f_{\partial}\right)^{2}-1\right)},
$$

где первое слагаемое характеризует дисперсию частотных флуктуаций в соответствии с [7]; второе слагаемое характеризует ГРК оценки частоты в канале с АБГШ в соответствии с [6]; $\tau$ - интервал измерения; $T_{\Pi}$ - период повторения измерений; $N$ - количество измерений; $R_{l}-$ ОСШ, выраженное в единицах; $f_{\partial}$ - частота дискретизации.

\section{Б. Расчет ГРК для белого фазового шума}

Как было отмечено выше, ГРК для случая белого фазового шума может быть рассчитана аналитически. Положим в (1) $\varphi[n]$ центрированным нормальным процессом с дисперсией $\Delta=\sigma_{\phi}^{2}$. Для малых значений фазовых флуктуаций в соответствии с [5] модель (1) можно переписать в виде

$$
x[n] \cong A(1+j \varphi[n]) \exp \left(j\left[2 \pi f_{0} n+\varphi_{0}\right]\right)+\dot{w}[n] .
$$

Воспользовавшись результатами Свинглера [6], ГРК можно записать в виде

$$
\operatorname{var}(\hat{f}) \cong \frac{6\left(2 R_{l} \Delta+1\right.}{(2 \pi)^{2} R_{l}\left(2 R_{l} \Delta^{2}+1\right)\left(N^{3}-N\right)} .
$$

Отметим, что оценка (5) при высоких уровнях ПОФ становится заниженной. Это связано с тем, что используемые при ее расчете формулы приближенного вычисления тригонометрических функций дают большую ошибку при больших значениях аргумента.

Другим путем получения приближенного выражения ГРК является представление аддитивного шума в виде белого фазового шума. В $[8,9]$ показано, что при высоких ОСШ модель (1) можно записать в виде

$$
x[n] \cong A \exp j\left[2 \pi f_{0} n+\varphi_{0}+\varphi[n]+\eta[n]\right],
$$

где $\eta[n]$ - центрированный нормальный процесс с дисперсией $\sigma_{\eta}{ }^{2}=\sigma_{w}^{2} / 2 A^{2}$.

Тогда оценку ГРК можно получить, представив фазовую функцию в виде процесса

$$
\phi[n]=2 \pi f n+\varphi_{0}+\varphi[n]+\eta[n] .
$$

Приняв допущение о независимости $\varphi[n]$ и $\eta[n]$, выразим функцию правдоподобия и информационную матрицу Фишера: 


$$
\begin{gathered}
L\left(\phi \mid f, \varphi_{0}\right)=\frac{1}{\left(2 \pi \sigma^{2}\right)^{\frac{N}{2}}} \exp \left(-\frac{1}{2 \sigma^{2}} \sum_{n=0}^{N-1}\left(\phi[n]-2 \pi f n-\varphi_{0}\right)^{2}\right) \\
I(\Theta)=\left[\begin{array}{c}
-E\left[\frac{\partial^{2} \ln L\left(\phi \mid f, \varphi_{0}\right)}{\partial f^{2}}\right]-E\left[\frac{\partial^{2} \ln L\left(\phi \mid f, \varphi_{0}\right)}{\partial f \partial \varphi_{0}}\right] \\
-E\left[\frac{\partial^{2} \ln L\left(\phi \mid f, \varphi_{0}\right)}{\partial f \partial \varphi_{0}}\right] \\
-E\left[\frac{\partial^{2} \ln L\left(\phi \mid f, \varphi_{0}\right)}{\partial \varphi_{0}^{2}}\right]
\end{array}\right]=\frac{1}{\sigma^{2}}\left[\begin{array}{cc}
2 \pi \sum_{n=0}^{N-1} n^{2} & 2 \pi \sum_{n=0}^{N-1} n \\
2 \pi \sum_{n=0}^{N-1} n & N]
\end{array}\right]= \\
=\frac{1}{\sigma^{2}}\left[\begin{array}{cc}
\frac{N(N-1)(N-2)}{6} & \frac{N(N-2)}{2} \\
\frac{N(N-2)}{2} & N]
\end{array}\right],
\end{gathered}
$$

где $\sigma^{2}=\sigma_{\eta}^{2}+\sigma_{\varphi}^{2}$.

Найдем обратную матрицу Фишера $I^{-1}(\Theta)$, и получим ГРК:

$$
\operatorname{var}(\hat{f}) \cong \frac{12\left(\sigma_{\eta}^{2}+\sigma_{\varphi}^{2}\right)}{(2 \pi)^{2} N\left(N^{2}-1\right)}=\frac{6\left(1+2 R_{l} \sigma_{\varphi}^{2}\right)}{(2 \pi)^{2} R_{l} N\left(N^{2}-1\right)},
$$

где $R_{l}=\frac{A^{2}}{\sigma_{w}^{2}}-$ ОСШ.

В отсутствии фазового шума выражение (6) вырождается в ГРК на выходе канала с АБГШ [6], а при малом уровне ПОФ это выражение очень близко к результатам Свинглера (5).

\section{3. РЕЗУЛЬТАТЫ МОДЕЛИРОВАНИЯ}

Результаты сравнения СКО алгоритма (2) с критерием (4) при воздействии процессов в соответствии с таблицей 1 представлены на рисунках 1-5. Размерность выборки $\mathrm{N}=2048$, количество экспериментов $\mathrm{K}=10000$. Моделирование фазового шума выполнялось методом с учетом спектральной маски фазового шума, описанным в $[10,11]$. ПОФ определялось по формуле

$$
\sigma_{\varphi}\left(f_{H}, f_{d}\right)=\sqrt{\int_{f_{H}}^{f_{d}} S_{\varphi}(f) d f}
$$

где $f_{d}$ - частота дискретизации; $f_{H}=1 / T, T$ - полный интервал наблюдения.

На рис. 1-3: 1-5 - кривые СКО исследуемого алгоритма для ПОФ $90^{\circ}, 45^{\circ}, 20^{\circ}, 10^{\circ}, 5^{\circ}$ соответственно, 6-10 теоретические границы в соответствии с (4), 11 - кривая ГРК в отсутствии фазового шума.

На рис. 4, 5: 1-4 - кривые СКО исследуемого алгоритма для ПОФ $20^{\circ}, 10^{\circ}, 5^{\circ}, 1^{\circ}$ соответственно, 5-8 теоретические границы в соответствии с (4), 9 - кривая ГРК в отсутствии фазового шума. 


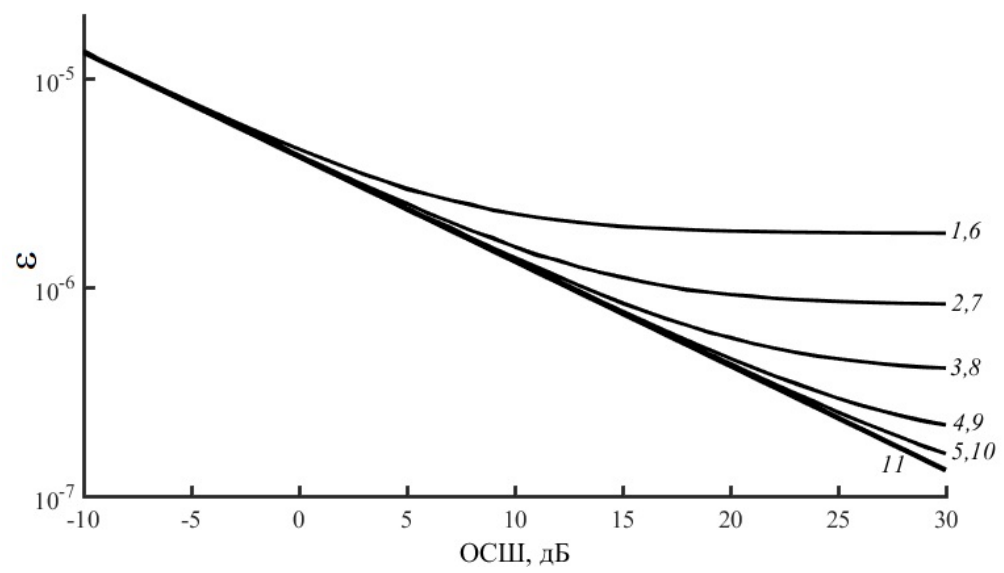

Fig. 1. The dependence of the standard error on the OSH and POF. Frequency noise of random walks

Рис. 1. Зависимости СКО от ОСШ и ПОФ. Частотный шум случайных блужданий

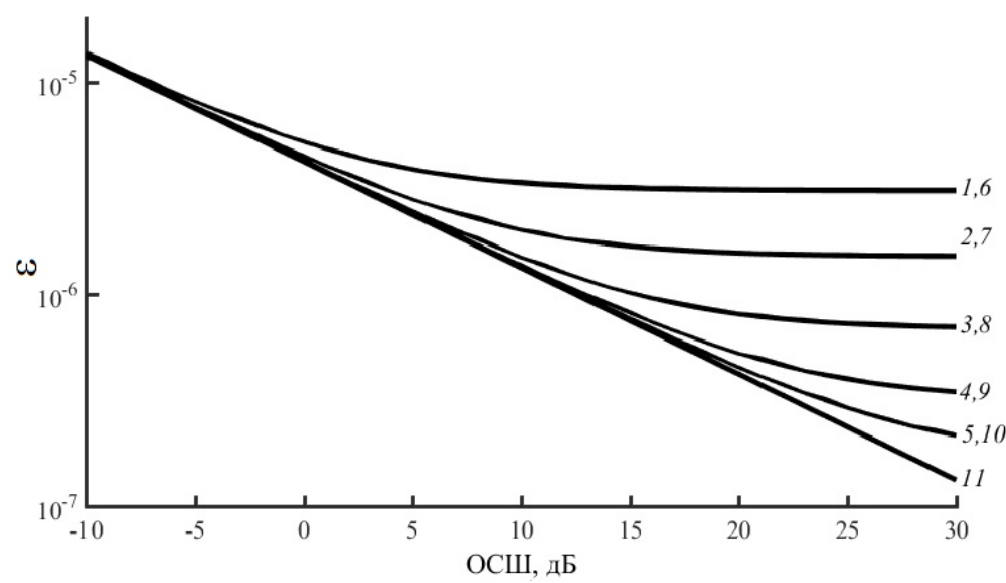

Fig. 2. The dependence of the standard error on the OSH and POF. Frequency flicker-noise

Pис. 2. Зависимости СКО от ОСШ и ПОФ. Частотный фликкер-шум

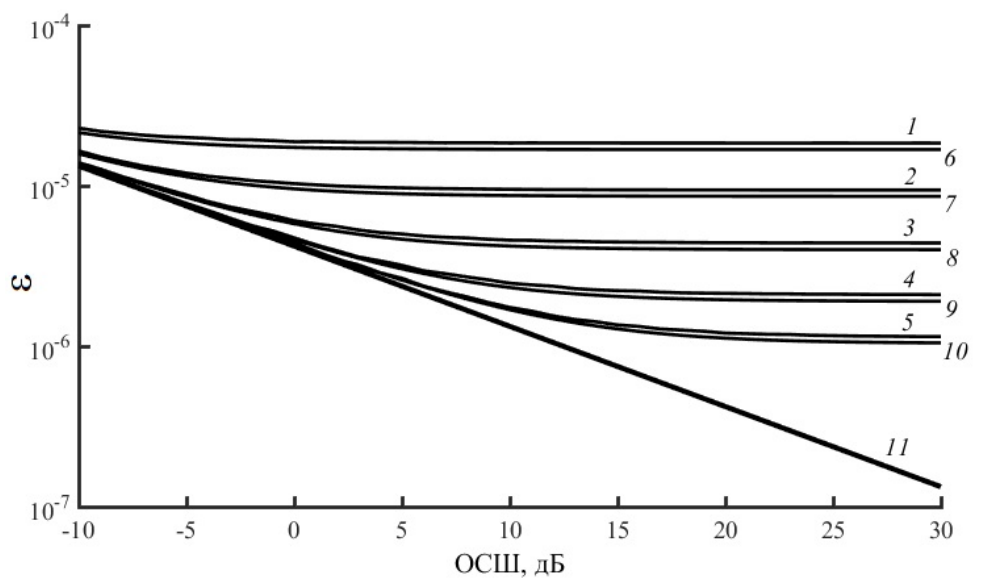

Fig. 3. The dependence of the standard error on the OSH and POF. White frequency noise
Рис. 3. Зависимости СКО от ОСШ и ПОФ. Белый частотный шум 


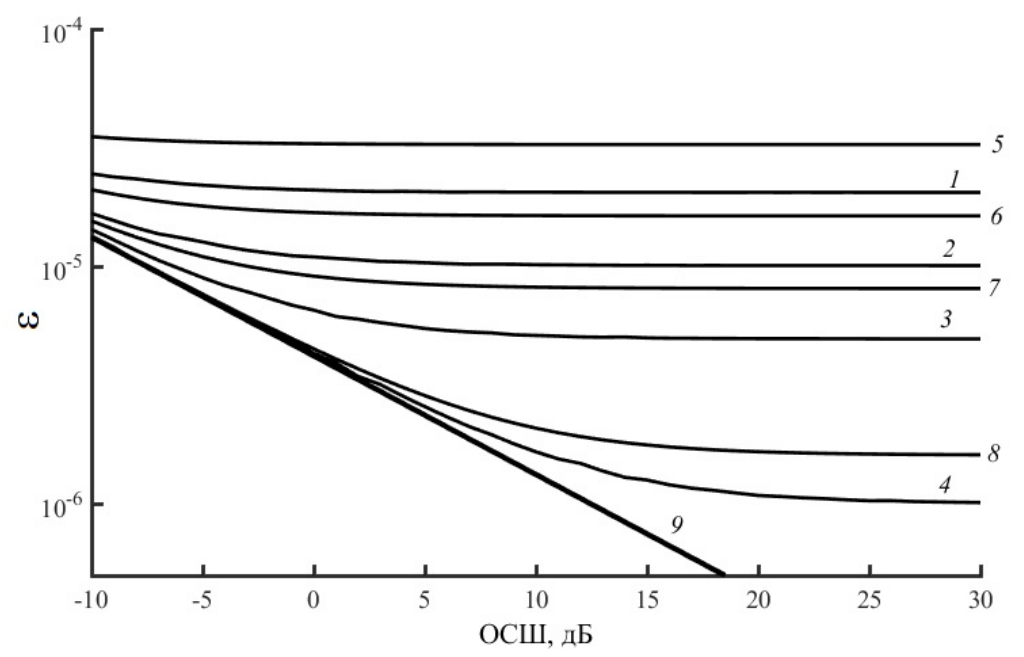

Fig. 4. The dependence of the standard error on the OSH and POF. Phase flicker-noise

Pис. 4. Зависимости СКО от ОСШ и ПОФ.

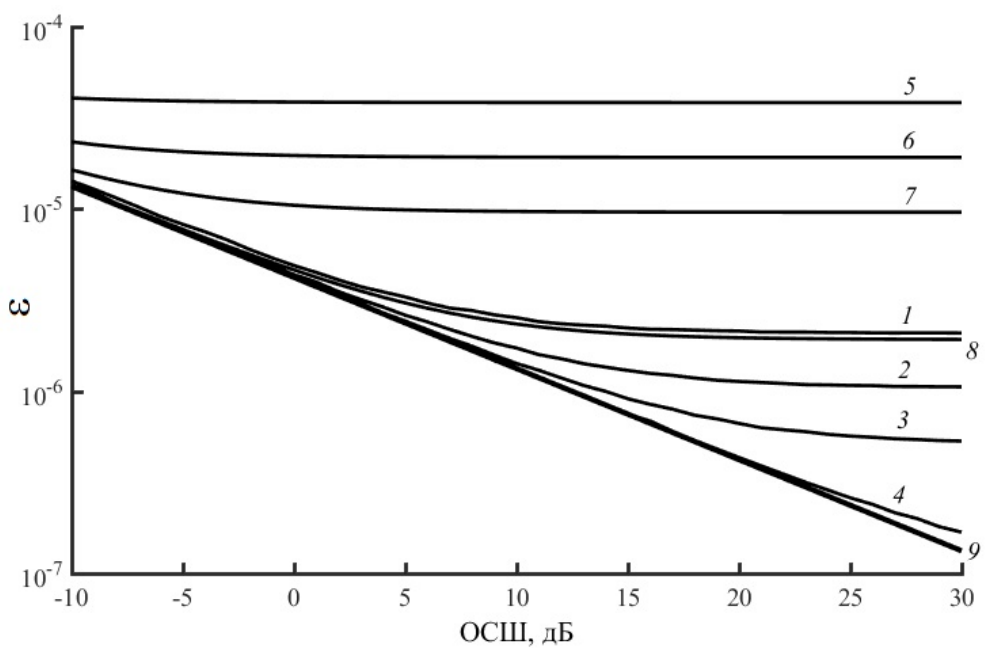

Fig. 5. The dependence of the standard error on the OSH and POF. White phase noise

Pис. 5. Зависимости СКО от ОСШ и ПОФ. Белый фазовый шум 
По рис. 4-5 можно сделать следующие выводы:

- в канале с частотными шумами СКО алгоритма близка к значениям по (4);

- в канале с фазовыми шумами оценка (4) является завышенной к СКО алгоритма. Это связано с тем, что (4) не является теоретической предельной границей. Поскольку ГРК для канала с белым фазовым шумом получена аналитически (выражения (5), (6)) целесообразно провести сравнение алгоритма с этими выражениями.

Результаты сравнения СКО алгоритма с выражениями (5) и (6) представлены на рис. 6, 7.

На рис. 6: 1-5 - кривые СКО исследуемого алгоритма для ПОФ $15^{\circ}, 10^{\circ}, 5^{\circ}, 2^{\circ}, 1^{\circ}$ соответственно, 6-10 теоретические границы в соответствии с (5), 11 - кривая ГРК в отсутствии фазового шума.

На рис. 7: 1-5 - кривые СКО исследуемого алгоритма для ПОФ $15^{\circ}, 10^{\circ}, 5^{\circ}, 2^{\circ}, 1^{\circ}$ соответственно, 6-10 теоретические границы в соответствии с (6), 11 - кривая ГРК в отсутствии фазового шума.

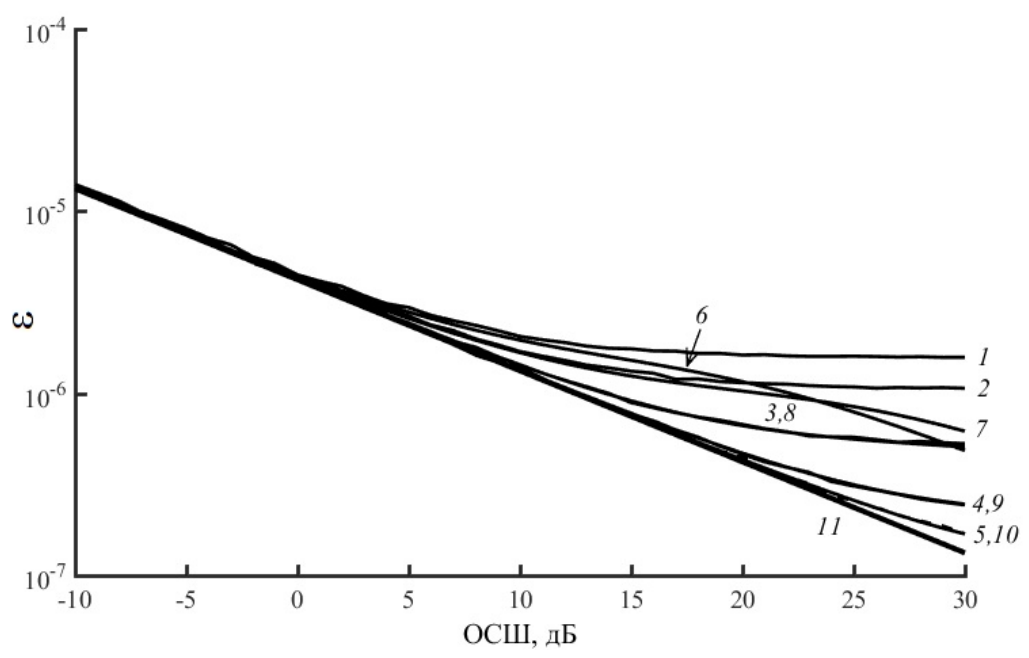

Fig. 6. The dependence of the standard error on the OSH and POF. White phase noise
Рис. 6. Зависимости СКО от ОСШ и ПОФ. Белый фазовый шум

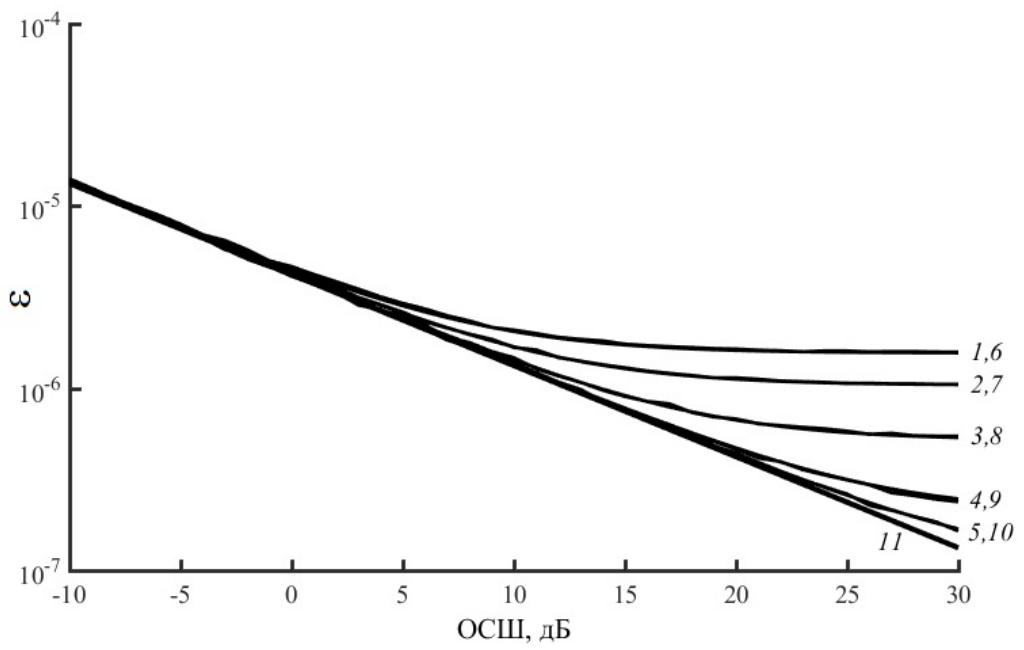

Fig. 7. The dependence of the standard error on the OSH and POF. White phase noise
Рис. 7. Зависимости СКО от ОСШ и ПОФ. Белый фазовый шум 
По представленным на рис. 6, 7 кривым видно, что при малых значениях ПОФ СКО алгоритма очень близка к рассмотренным приближениям ГРК. Однако, как было отмечено выше, при высоких уровнях ПОФ граница, полученная Свинглером становится заниженной. Выражение (6) дает более точное приближение.

\section{Выводы}

1. Представлены выражения, приближенно описывающие СКО алгоритма оценки частоты, основанного на итерационном вычислении АКП, в условиях частотных шумов и АБГШ. На основании этих выражений алгоритм может использоваться для анализа долговременной частотной нестабильности коротких импульсно-модулированных сигналов.

2. Предложена формула приближенного вычисления ГРК в условиях белого фазового шума. Результаты моделирования показали, что предложенная формула точнее описывает СКО алгоритма при высоких значениях ПОФ, чем известная формула Свинглера.

3. В канале с белым фазовым шумом и АБГШ алгоритм, основанный на итерационном вычислении АКП обеспечивает оценку с СКО близкой к границе Рао-Крамера.

\section{References}

[1] A. A. Nikiforov, "Identification and assessment of information parameters of navigation systems with code division", in Thesis for the degree of candidate of technical sciences, In Russian, Bauman Moscow State Technical University, 2014.

[2] V. G. Volkov, Y. N. Krivov, and I. V. Lukyanov, "Improved frequency estimation algorithm based on iterative computation of autocorrelation sequence”, fournal of Radio Electronics, no. 10, 2016, In Russian. [Online]. Available: http://jre.cplire.ru/jre/oct16/6/text.html.

[3] A. V. Ryzhkov and V. N. Popov, Frequency synthesizers in radio communication technique. Moscow: Radio and Communication, 1991, p. 264, In Russian.

[4] W. J. Riley, Handbook of Frequency Stability Analysis. USA - Washington: National Institute of Standards and Technology, 2008.

[5] D. N. Swingler, "Approximations to the Cramer-Rao lower bound on frequency estimates for complex sinusoids in the presence of sampling jitter”, Signal Processing, vol. 48, no. 1, pp. 77-83, 1996.

[6] S. M. Kay, Fundamentals of Statistical Signal Processing: Estimation Theory (v.1). NJ: Prentice Hall, Upper Sadle River, 1998, p. 595.

[7] J. A. Barnes et al., "Characterization of frequency stability", in IEEE Transactions on Instrumentation and Measurement, vol. IM-20, 1971, pp. 105-120.

[8] A. Tretter, "Estimating the Frequency of a Noisy Sinusoid by Linear Regression", IEEE Transactions on Information theory, vol. 31, no. 6, pp. 832-835, 1985.

[9] S. M. Kay, "A Fast and Accurate Single Frequency Estimator", IEEE Transactions on Acoustics, Speech, and Signal Processing, vol. 37, no. 12, pp. 1987-1990, 1989.

[10] Y. V. Kryukov, E. V. Rogozhnikov, and D. A. Pokamestov, "Phase noise model taking into account the spectral mask of frequency synthesizers and signal generators", Bulletin of the Tomsk Polytechnic University. Information Technology, vol. 325, no. 5, pp. 45-51, 2014, In Russian.

[11] J. A. Barnes, "Atomic Timekeeping and the Statistics of Precision Signal Generators", Proceeding of the IEEE, vol. 54, no. 2, pp. 207-220, 1966. 